\title{
Numerical Simulation Analysis of Influence of Explosive Performance on JPC Molding
}

\author{
Kui-wen Cuil, a , Shuang-shan $\mathrm{Mi}^{1}$ \\ ${ }^{1}$ School of Ordnance Engineering College, Shijiazhuang 050003, China; \\ accuikuiwen@126.com
}

\begin{abstract}
Keywords: Explosive material, Jetting Projectile Charge, molding parameters, numerical simulation,
\end{abstract} simulation verification.

\begin{abstract}
In order to study the impact of explosive performance on JPC molding, simulation software of AUTODYN-2D is used in JPC molding. Numerical simulation analysis of influences of five different explosives on JPC molding is performed. Method of determining the molding time is proposed. Two main results can be concluded. First, explosive material has no influence on JPC molding time. Under the same charge structure, JPC molding time of different explosive material has no difference. Second, the impact laws of explosive density and detonation velocity on JPC molding speed, fineness ratio and other molding parameters are proposed. The greater the density and detonation velocity of the explosive is, the better the performance of JPC molding parameters is and also the more conducive to the penetration of JPC, last verify the correctness and reliability of the laws by simulation test, the results provide a reference for rod shaped penetration warhead.
\end{abstract}

\section{Introduction}

Three kinds of penetrators were developed in shaped charge field according to cone angle of the liner. They are JET, Jetting Projectile Charge (JPC), and Explosively Formed Projectile (EFP) [1]. JET is in deep penetration, but aperture is small. The aperture of EFP is large, but penetration depth is small. The performance of JPC is between JET and EFP. The penetration depth of JPC is smaller than JET, but aperture is bigger, and flight distance is long. JPC has much bigger fineness ratio and better continuity, and is not easy to be broken. With the appearance of the new type of reactive armor and active defense, the protection ability of the modern armored target has been greatly improved. The JPC with good continuity and strong penetration ability has a good application prospect in the field of anti-armor warhead under heavy bombing condition, and becomes the new popular research target.

The impact factors on JPC molding are detonation ways, medicine type cover material, geometric structure and explosive material, etc. Ya-dong Yang studied the impact of detonation ways on JPC molding [2]. Han-ling Wu studied the whole process of medicine type cover pressing cross, the formation of the jet, the extension of the jet, and the unstable fracture, and analyzed the material and structure of medicine type cover[3]. Liang Zhu studied the impact of explosive material performance on JPC molding, but didn't perform study on JPC molding time. There are many researches on JPC molding at home and abroad, but fewer studies the impact of explosive material on JPC molding [4]. Based on the existing research, this paper proposes the methods of determining JPC molding time. AUTODYN simulation software is used in this paper to analyze the impacts of the density and detonation velocity of TNT、COMP B、8701、PBX9501, and HMX on JPC molding time and fineness ratio. The results provide a solution to difficulties of selecting explosives in the process of JPC application.

\section{Finite element model establishment}

\subsection{Computational model}

Numerical simulation is flexible and repeatable, and has gradually become a kind of economical and effective numerical test method. The most frequently-used explosion dynamics numerical simulation software is AUTODYN and LS-DYNA at home and abroad. The software can effectively simulate a 
series of problems about shaped charge. AUTODYN is used in this paper on studying shock initiation to reduce computational error. Rich material models and corresponding parameters are embedded in AUTODYN software, including various types of metal, ceramic materials, glass, cement, all kinds of explosives, water, air and other solid, fluid and gas materials model and corresponding data [5]. There is also a variety of algorithms that are embedded in AUTODYN software, and the core of any simulation software is election of correct algorithm. The substance of JPC molding is explosion and shock problems. The root problem is the of multi material fluid elastic plastic dynamics. Therefore, the algorithm can be divided into Lagrange algorithm and Euler algorithm in terms of coordinate problems.

Lagrange algorithm. Under Lagrange algorithm, the material is attached to the grid, that is, the grid is moving along with the material. Small solid materials, moderate deformation can be better simulated using Lagrange algorithm. Compared with other algorithm, Lagrange algorithm has quicker speed and higher accuracy, but when the deformation of the object is too large, it will lead to excessive distortion of the grid and even distortion, which will lead to a decline in the accuracy of the calculation and cannot continue to be calculated. Therefore, this method has good adaptability and accuracy for the simulation of penetration, collision type low speed, and moderate deformation problems.

Euler algorithm. Different from Lagrange algorithm, the coordinate of Euler algorithm is a fixed spatial coordinate system. The material flows in the grid, that is, the mesh does not deform with the material movement. The algorithm is suitable for describing the behavior of liquid and gas, especially for simulating large deformation problems, because it does not involve the deformation of the grid. The disadvantage is that the complex algorithm must be used to track the motion of the material, so the calculation speed is very slow.

JPC molding is a kind of deformation motion with multi-matter integration. More and more intense deformation of explosive material and liner material will appear in the process of metal jet formation. After analyzing the characteristics of the two algorithms, it is difficult to accurately simulate using Lagrange. Mess deformation is not involved in Euler algorithm, therefore Euler algorithm is suitable for study of explosions and other large deformation. Euler algorithm can accurately describe the process of JPC molding. Therefore, Euler algorithm is used in this paper.

The main charge, shell, liner and air fields are built using the Euler unit. In view of the shortcomings of slow computation speed using Euler algorithm, the numerical simulation set a large model grid, a total of $1000 \times 50$ micro- elements, and refine the mesh in the previous section needed to be observed of the model [6]. Refine the mesh can make the size of the jet formation area reasonable that not only guarantees the accuracy of the simulation but also reduces the simulation running time reasonably. The boundary condition of the model is Flow-Out which is no reflection, and the reflection of the detonation wave is prevented from affecting the JPC formation. As it relates to shock initiation problems, the unit system is cm-g- $\mu \mathrm{s}$.

\subsection{Geometric model}

The shaped charge is stern-shaped structure with central initiation, and the liner is designed as arc cone combined structure. The structure diagram of the shaped charge ( $1 / 2$ sectional view) is depicted in Fig. 1.

Because the shaped charge is axisymmetric, the model is built with $1 / 4$ structure that there is no impact on the authenticity of the simulation of authenticity. It can reduce the amount of computation to reduce the simulation time, and it also reduces the errors of accumulation in the simulation operation and improves the reliability and stability of the simulation. 


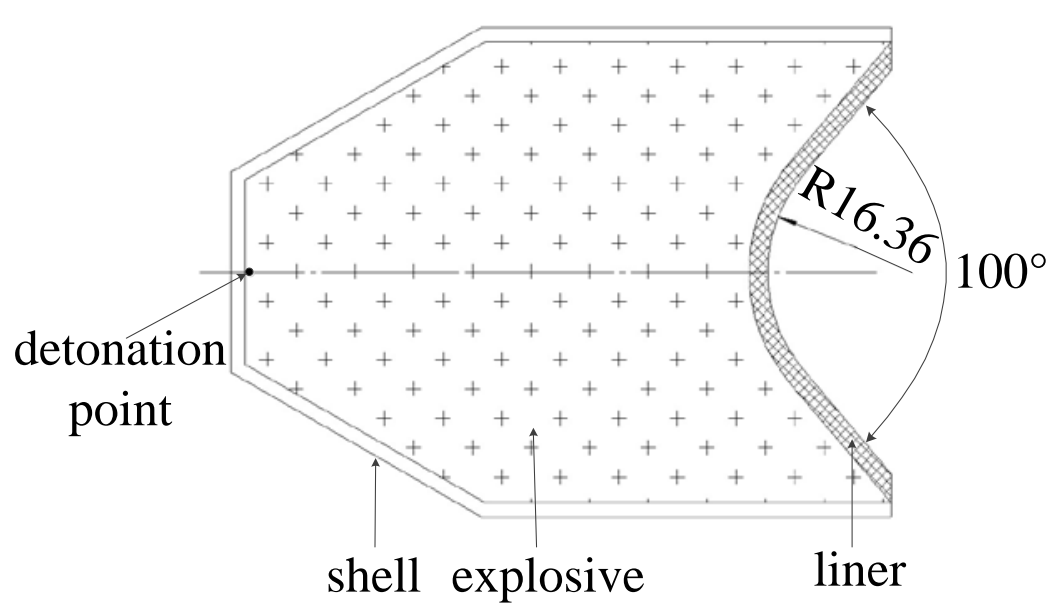

\subsection{Material model}

Fig. 1 Schematic diagram of shaped charge structure

Rich material models are embedded in AUTODYN software, and the material model is mainly composed of state equation, strength model and failure model [5]. The numerical simulation of the impact of explosives on JPC molding involves explosives, air, shells and liners.

TNT, COMP B, 8701, PBX 9501, HMX are selected from the AUTODYN material library, and the equation of state is the standard JWL equation. The state equation is often used to describe explosives and detonation products, and it also can be used to calculate the chemical energy into the pressure, the relation of pressure and energy is shown as below [7].

$$
\rho=A_{1}\left(1-\frac{\omega}{R_{1} V}\right) e^{-R_{1} V}+B_{1}\left(1-\frac{\omega}{R_{2} V}\right) e^{-R_{2} V}+\frac{\omega E}{V}
$$

In formula (1), the $A_{1}, B_{1}, R_{1}, R_{2}$ and $\omega$ are the material constants, $p$ is the pressure, $V$ is the relative volume, $E$ is the initial internal energy. The relevant parameters are shown in Table 1.

Table 1 The main parameters of explosive materials

\begin{tabular}{cccccccccc}
\hline Material & $\begin{array}{c}\rho \\
\mathrm{g} \cdot \mathrm{cm}^{-3}\end{array}$ & $\begin{array}{c}D \\
\mathrm{~m} \cdot \mathrm{s}^{-1}\end{array}$ & $\begin{array}{c}A_{1} \\
\mathrm{GPa}\end{array}$ & $\begin{array}{c}B_{1} \\
\mathrm{GPa}\end{array}$ & $R_{1}$ & $R_{2}$ & $\omega$ & $\begin{array}{c}E \\
\mathrm{GPa}\end{array}$ & $P$ \\
\hline TNT & 1.630 & 6930 & 373.77 & 3.7470 & 4.15 & 0.90 & 0.35 & 6.00 & 21.00 \\
COMP B & 1.717 & 7980 & 524.23 & 7.6780 & 4.20 & 1.10 & 0.34 & 8.50 & 29.50 \\
8701 & 1.780 & 8425 & 854.50 & 20.4930 & 4.60 & 1.35 & 0.25 & 9.50 & 29.66 \\
PBX9501 & 1.840 & 8800 & 852.40 & 18.0200 & 4.55 & 1.30 & 0.38 & 10.20 & 37.00 \\
HMX & 1.891 & 9110 & 778.28 & 7.0714 & 4.20 & 1.00 & 0.30 & 10.50 & 42.00 \\
\hline
\end{tabular}

The model needs to establish the air field. The material is "AIR" with the initial internal energy. The shell is made of polyethylene. The corresponding material in the software library is "POLYETHYL". The material of the liner is copper, the corresponding material in the software is "COPPER", at the same time, change the material's yield strength "Strength" to "Johnson-Cook" form as required [8].

\section{Numerical simulation results and analysis}

The forming parameters of the penetrating body are determined by the detonation energy produced by the detonation of the explosive. According to the theory of detonation, the detonation energy of explosives mainly depends on the material properties such as density and detonation velocity. Determining the impact law of the density of and detonation velocity of the explosives on JPC forming by numerical simulation has an important significance on optimizing the warhead penetrating body's performance and improving the penetrating ability. 


\subsection{Determination of JPC molding time}

In order to obtain the parameters of JPC molding, it is necessary to provide a reference for future research. Firstly, the standard forming time should be unified. The observed parameters have reference significance only under the uniform standard. If the JPC molding time chosen is too small, the jet is not sufficiently stretched to adequately reflect its performance. If time is too large, JPC can maintain continuous and stable in a long time. It is difficult to unify the standard observation time, as the JPC head-to-tail velocity gradient exists. The fineness ratio and other parameters of the JPC will continue to change. There are different results in different observation time. Therefore, this paper proposes that the time when the head speed does not increase rapidly and remains stable is JPC molding time. In order to determine the molding time of JPC, continuous and equidistant fixed gauges are added on the model as shown in Fig. 2.

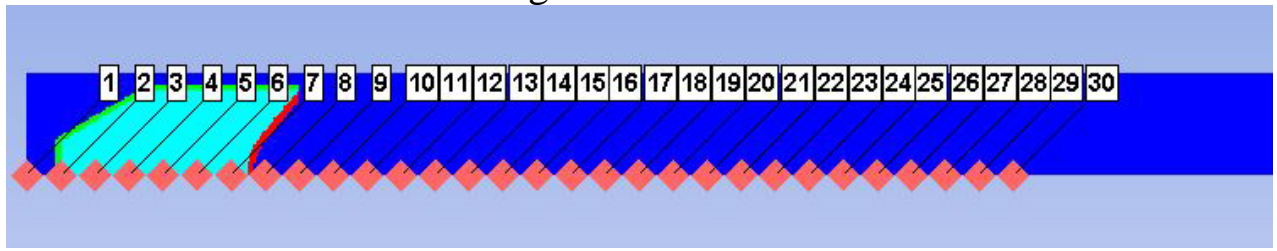

Fig. 2 Fixed guages

Speed cloud of the fixed gauges is depicted in Fig. 3. It can be seen from Fig.3 that the trend of the gauges' peak velocity remain stable at $t=30 \mu$ s. It shows that the head speed of JPC is stable after $t=30 \mu \mathrm{s}$. And the JPC forming time with five different materials is $t=30 \mu \mathrm{s}$, it shows that the explosive material does not affect JPC molding time.

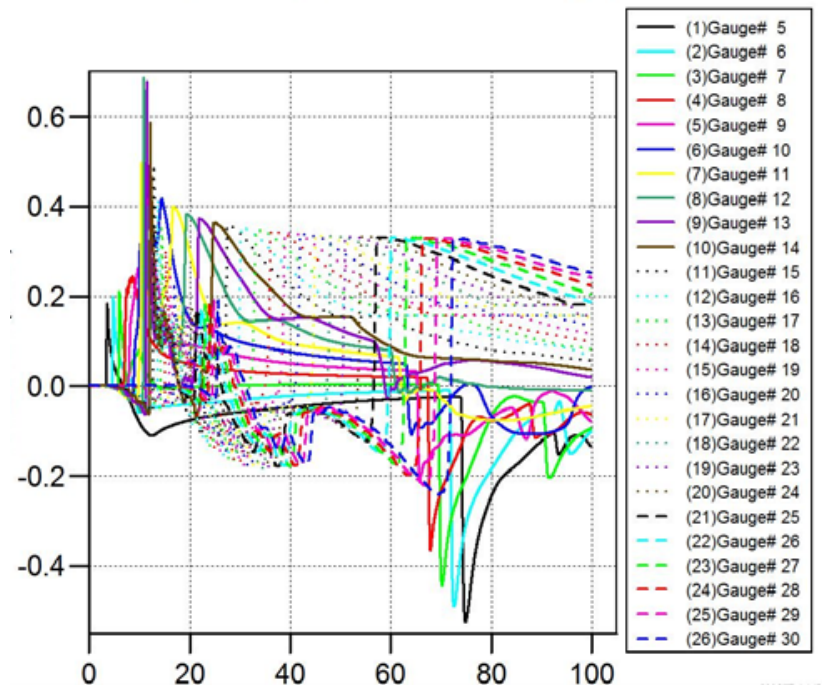

Fig. 3 Speed cloud of the fixed gauges

\subsection{JPC molding parameters}

It can be obtained from 3.1 that the molding time is $t=30 \mu \mathrm{s}$. The model diagram of the JPC molding with different explosive materials is depicted in Fig. 4.
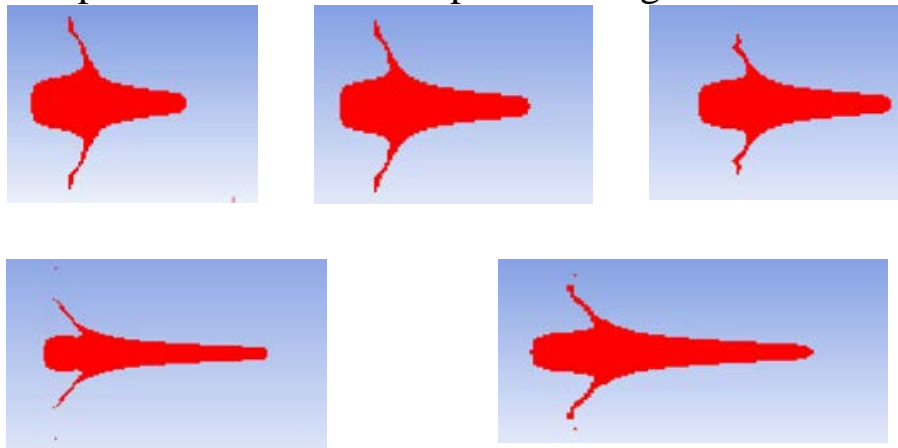

Fig. 4 Diagram of JPC molding with different explosive materials 
The head speed, tail speed, length, and diameter and other data can be obtained from the software of AUTODYN after JPC molding time. And the relevant parameters are shown in Table 2.

Table 2 JPC molding parameters

\begin{tabular}{ccccccc}
\hline Material & $\begin{array}{c}\text { Head speed } \\
\mathrm{m} \cdot \mathrm{s}^{-1}\end{array}$ & $\begin{array}{c}\text { Tail speed } \\
\mathrm{m} \cdot \mathrm{s}^{-1}\end{array}$ & $\begin{array}{c}\mathrm{H}-\mathrm{T} \text { difference } \\
\mathrm{m} \cdot \mathrm{s}^{-1}\end{array}$ & $\begin{array}{c}\text { Length } \\
\mathrm{cm}\end{array}$ & $\begin{array}{c}\text { Diameter } \\
\mathrm{cm}\end{array}$ & $\begin{array}{c}\text { Fitness } \\
\text { ratio }\end{array}$ \\
\hline TNT & 2983 & 1212 & 1771 & 3.72 & 1.32 & 2.8182 \\
COMP B & 3184 & 1292 & 1892 & 4.32 & 1.28 & 3.3750 \\
8701 & 3494 & 1405 & 2089 & 4.86 & 1.20 & 4.0500 \\
PBX9501 & 3520 & 1416 & 2104 & 4.92 & 1.14 & 4.3158 \\
HMX & 3549 & 1378 & 2171 & 5.04 & 1.08 & 4.6667 \\
\hline
\end{tabular}

\subsection{Impact Law of density Explosive on JPC Molding}

Table 2 shows that when the explosive density increases from $1.630 \mathrm{~g} \cdot \mathrm{cm}^{-3}$ to $1.891 \mathrm{~g} \cdot \mathrm{cm}^{-3}$, the head speed increases by $15.6 \%$ and the fitness ratio increases by $65.6 \%$. The overall analysis of five explosives show the relationship between the head speed and the fitness ratio of JPC with the explosive density, and the fitting curve is obtained by using Origin. The relationship of the fitness varies with density is depicted in Fig. 5, and the relationship of the head speed varies with density is depicted in Fig. 6.

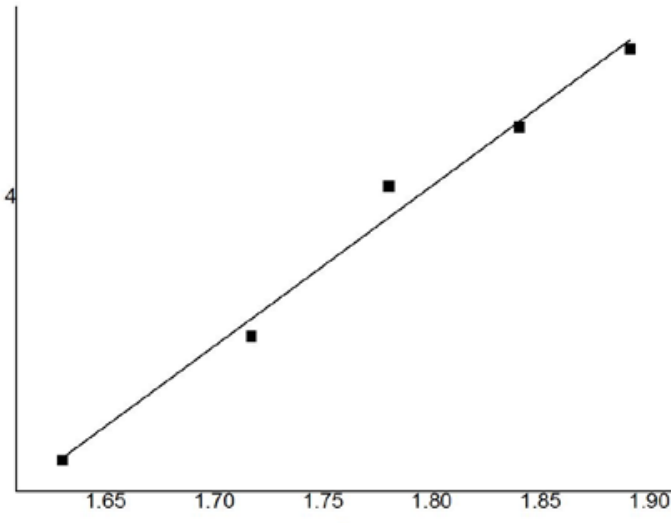

Fig.5 Curve of fitness ratio with density The equation is as follows:

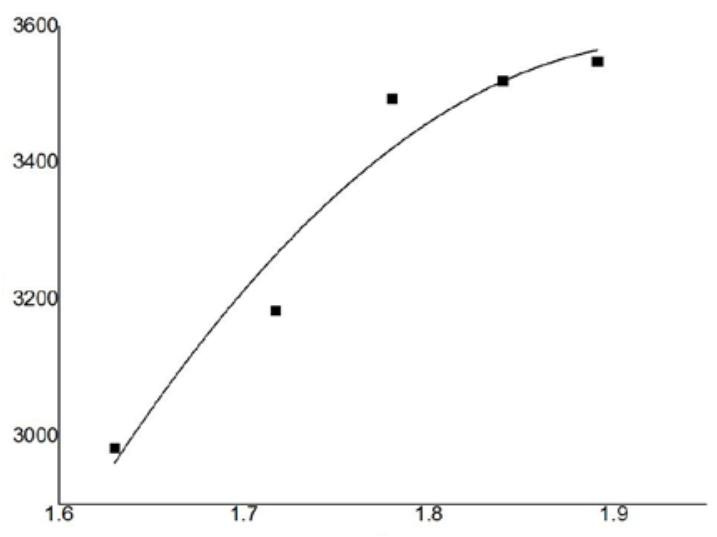

Fig.6 Curve of head speed with density

$$
\begin{aligned}
& L / D=-8.92828+7.2101 \rho \\
& V=-21797.13591+26285.40283 \rho-6807.83672 \rho^{2}
\end{aligned}
$$

In the formula, $L / D$ is the fitness ratio; $\rho$ is the explosive density; $V$ is the JPC head speed.

It can be drawn from Table 2, Figure 5 and Figure 6 that JPC molding head speed and fitness ratio increase with the increase of density, when the explosive density $\rho>1.630 \mathrm{~g} \cdot \mathrm{cm}^{-3}$.

\subsection{Impact Law of detonation velocity of explosive on JPC molding}

The detonation velocity of the explosive is an important parameter of the explosive material, and it's also an important factor on JPC molding. By analyzing the case of JPC molding under different explosive materials, the fitting curve and the fitting equation of the fitness ratio of the penetration body and the velocity of the head with the detonation velocity can be obtained, and they are depicted in Fig.7 and Fig. 8. 


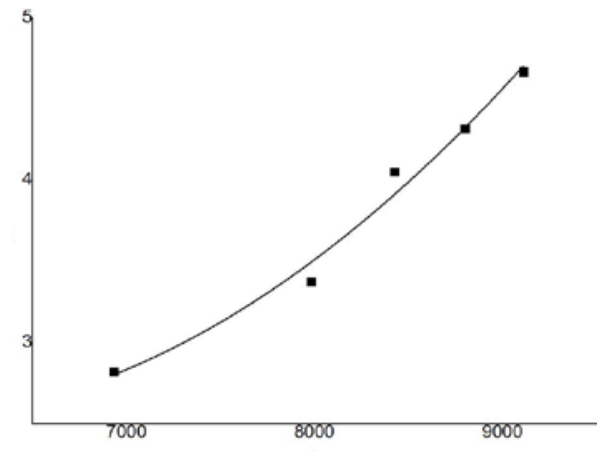

Fig.7 Curve of fitness ratio with detonation velocity

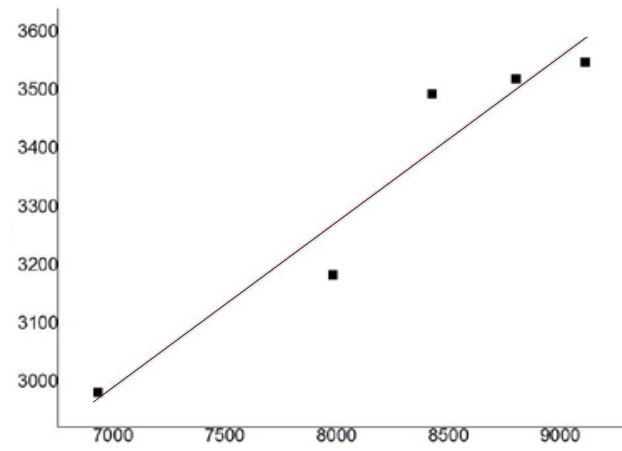

Fig.8 Curve of head speed with detonation velocity

The equation is as follows:

$$
\begin{aligned}
& L / D=8.64139-0.00215 D_{1}+1.88217 \times 10^{-7} D_{1}^{2} \\
& V=1000.47641+0.28361 D_{1}
\end{aligned}
$$

In the formula, $L / D$ is the fitness ratio; $D_{1}$ is the detonation velocity of explosive; $V$ is the JPC head speed.

It can be drawn from Table 2, Figure 7 and Figure 8 that JPC molding head speed and fitness ratio increase with the increase of detonation velocity when the detonation velocity $D_{1}>6930 \mathrm{~m} \cdot \mathrm{s}^{-1}$.

\section{Simulation verification}

In order to verify the accuracy of the impact law of the density and detonation velocity of explosive on the fitness and head speed of JPC, select LX-14 for simulation and verification, and the main parameters of LX-14 are shown in Table 3.

Table 3 Relevant parameters of LX-14

\begin{tabular}{cccccccccc}
\hline Material & $\begin{array}{c}\rho \\
\mathrm{g} \cdot \mathrm{cm}^{-3}\end{array}$ & $\begin{array}{c}D \\
\mathrm{~m} \cdot \mathrm{s}^{-1}\end{array}$ & $\begin{array}{c}A_{1} \\
\mathrm{GPa}\end{array}$ & $\begin{array}{c}B_{1} \\
\mathrm{GPa}\end{array}$ & $R_{1}$ & $R_{2}$ & $\omega$ & $\begin{array}{c}E \\
\mathrm{GPa}\end{array}$ & $P$ \\
\hline LX-14 & 1.835 & 8800 & 826.1 & 17.24 & 4.55 & 1.32 & 0.38 & 10.2 & 37 \\
\hline
\end{tabular}

It can be obtained from LX-14 speed cloud of the gauges that the molding time of JPC is still $t=30 \mu \mathrm{s}$. It verifies that the explosive material has no influence on the forming time of JPC. Then parameters of JPC molding are observed at $t=30 \mu \mathrm{s}$, and comparison of parameters observed and the theoretical parameters obtained by the law is shown in the Table 4.

Table 4 Comparison of molding parameters observe and theoretical parameters

\begin{tabular}{ccccc}
\hline LX-14 & $\begin{array}{c}\text { Head speed } \\
\mathrm{m}^{-1} \mathrm{~s}^{-1}\end{array}$ & $\begin{array}{c}\text { length } \\
\mathrm{cm}\end{array}$ & $\begin{array}{c}\text { diameter } \\
\mathrm{cm}\end{array}$ & Fitness ratio \\
\hline Observations & 3474 & 4.88 & 1.14 & 4.2807 \\
Theoretical value & 3513 & & & 4.3026 \\
Error(\%) & 1.1226 & & & 5.1160
\end{tabular}

It can be drawn from Table 4 that the error between the results obtained by empirical law and the observed results is small. The error is within a reasonable range, and the error of head speed is only $1.1226 \%$. It can be concluded that the simulation results are correct and reliable.

\section{Summary}

In order to study the influence of explosive material on JPC molding, the numerical simulation is carried out by AUTODYN software. In view of the current condition that there is no unified standard of JPC molding time, in order to make a more scientific and reasonable observation of the parameters 
after JPC molding, a method of determining JPC molding time is proposed after reasonable analysis and full use of the software. The parameters of JPC molding are obtained after forming the finite element model of JPC, and the data was processed by Origin software. The impact law of explosive density and detonation velocity on the fitness ratio and head speed of JPC is drawn. The conclusions are as follows:

(1) Explosive material does not affect the time of JPC molding. Simulation results show that, under the same conditions of charge structure, the time of JPC molding is unchanged when the explosive material is changed.

(2) As the explosive density increases, the fitness and head speed of the JPC penetrator are increasing. When the density of explosives increases from $1.630 \mathrm{~g} \cdot \mathrm{cm}^{-3}$ to $1.891 \mathrm{~g} \cdot \mathrm{cm}^{-3}$, the head speed of JPC is increased by $15.6 \%$ and the fitness ratio is increased by $65.6 \%$. And with the increase of detonation velocity, the fitness ratio of JPC increases steadily and the head speed increases linearly.

(3) The impact law of five explosives (TNT, COMP B, 8701, PBX9501 and HMX) on JPC molding are studied by simulation. And it is concluded that large density and high detonation velocity of explosive materials is helpful to improve the penetration efficiency of the JPC, which provides a reference for the application of JPC.

\section{References}

[1] Jian-ping Yin, Zhi-jun Wang, Ammunition Theory, first ed., Beijing Institute of Technology Press, Beijing, 2014.

[2] Ya-dong Yang, Zhi-gang Chen, Numerical Simulation of Jetting Penetrator Charge Formation under Different Initiation Ways, J. Journal of Projectiles, Rockets, Missiles and Guidance. 2008, 28 (5) 89-91,98.

[3] Han-ling Wu, Zhuo-ping Duan, Yong-qing Wang, Numerical Simulation of the Rod-liked Jet Formation, J. Sci. Explosion and Shock Waves. 2006, 26 (4) 328-332.

[4] Liang Zhu, Hui-zi Li, Xiao-ming Wang, Impact of Parameter in Explosive Material for JPC

Molding, J. Journal of Sichuan Ordnance. 2011, 32 (3) 13-16.

[5] Shao-qing Shi, Min Wang, Bo Sun, Xiang-wei Fang, Yingfang Liu, AUTODYN Engineering Dynamic Analysis and Application Examples, first ed., China Building Industry Press, Beijing, 2012.

[6] Jian-bin Men, Jian-wei Jiang, Shu-you Wang, Fundamentals of Numerical Simulation for Explosion and Shock Problems, first ed., Beijing Institute of Technology Press, Beijing, 2015.

[7] Zheng-xiang Huang, Theory and Practice of Shaped Charge, first ed., Beijing Institute of Technology Press, Beijing, 2014.

[8] Flis WJ, The Effects of finite liner Acceleration on Shaped Charge Jet Formation, C. 19th International Symposium of Ballistics. USA, International Ballistics Committee, 2011. 